\title{
Prostate Cancer Imaging and Therapy
}

Editors

RICHARD P. BAUM

CRISTINA NANNI

\section{PET CLINICS}

www.pet.theclinics.com

Consulting Editor

ABASS ALAVI

April 2017 - Volume 12 - Number 2 